\title{
Balkanologie
}

Balkanologie Revue d'études pluridisciplinaires

Vol. VII, $n^{\circ} 1$ | 2003

Volume VII Numéro 1

\section{Entre alliance et crise de confiance : la politique balkanique de la France et son échec (1938-1940)}

\section{Constantinos Prévélakis}

\section{(2) OpenEdition}

12 Journals

Édition électronique

URL : http://journals.openedition.org/balkanologie/477

DOI : $10.4000 /$ balkanologie.477

ISSN : 1965-0582

Éditeur

Association française d'études sur les Balkans (Afebalk)

Édition imprimée

Date de publication : 1 juin 2003

Pagination : 137-146

ISSN : 1279-7952

\section{Référence électronique}

Constantinos Prévélakis, «Entre alliance et crise de confiance : la politique balkanique de la France et son échec (1938-1940) », Balkanologie [En ligne], Vol. VII, n 1 | 2003, mis en ligne le 18 février 2009, consulté le 17 décembre 2020. URL : http://journals.openedition.org/balkanologie/477 ; DOI : https:// doi.org/10.4000/balkanologie.477 


\title{
ENTRE ALLIANCE ET CRISE DE CONFIANCE : LA POLITIQUE BALKANIQUE DE LA FRANCE ET SON ÉCHEC (1938-1940)
}

\author{
Constantinos Prévélakis*
}

Cet article constitue un état d'avancement des recherches d'une thèse en cours portant sur la complexe affaire des fronts d'Orient, dont la France fut le grand promoteur entre la conférence de Munich (octobre 1938) et l'entrée des troupes allemandes dans Paris (juin 1940) ${ }^{1}$. Les hypothèses de travail qui seront ici énoncées sont le fruit d'une première année d'études aux archives du Quai d'Orsay à Paris, du Service Historique de l'armée de Terre à Vincennes, ainsi que du Ministère hellénique des Affaires étrangères à Athènes ${ }^{2}$, focalisée principalement sur une grande puissance, la France, et deux puissances balkaniques, la Grèce et la Turquie.

Bien que situés en périphérie de l'Europe, les Balkans possèdent une place primordiale pour toute grande puissance qui entend élaborer une stratégie à l'échelle du continent, que ce soit en temps de guerre ou en temps de paix. À la croisée de trois continents, la péninsule balkanique contrôle les grandes voies de descente de l'Europe centrale et orientale vers les mers chaudes, ainsi que les voies de contact de l'Europe avec le Levant. En 1914 déjà, la Grande Guerre avait débuté dans cette région qui combinait de manière spectaculaire les ambitions contradictoires des acteurs du conflit : allemandes et autrichiennes sur

\footnotetext{
"Université de Paris IV-Sorbonne.

${ }^{1}$ Une synthèse plus détaillée a été rédigée dans le cadre d'un mémoire de DEA sous la direction du Professeur Georges-Henri Soutou intitulé La Grèce, la Turquie et les projets français de fronts d'orient 1938 1940, Université de Paris IV Sorbonne, juin 2000. La question avait été réellement évoquée pour la première fois par les historiens en 1969 lors du colloque organisé par le C.N.R.S. et intitulé La guerre en Méditerranée. Les actes furent publiés en 1971 par les éditions du C.N.R.S. à Paris.

${ }^{2}$ Ces fonds seront mentionnés en notes de bas de page sous les abréviations suivantes : MAE (archives du Ministère français des Affaires Etrangères), SHAT (Service Historique de l'Armée de Terre), EMA (EtatMajor de l'Armée), YPEX (Hypourgeion Exoterikon, Ministère Hellénique des Affaires Etrangères), AYE (Arheion tou Hypourgeiou Exoterikon, Archives du Ministère Hellénique des Affaires Etrangères).
} 
l'axe formé par les vallées du Danube, de son affluent Morava et du Vardar, russes sur Constantinople et les détroits du Bosphore et des Dardanelles, britanniques sur la Méditerranée orientale et le Levant, italiennes, enfin, sur la Dalmatie et l'Asie Mineure. Dès la première année du conflit, les Français et les Britanniques avaient tenté en vain de prendre les détroits, puis débarqué et constitué une tête de pont à Salonique, les puissances centrales avaient occupé la Serbie puis la Bulgarie et la Macédoine orientale, l'Italie s'était installée en Albanie et avait fait promettre, contre son entrée en guerre aux côtés de l'Entente, l'Istrie, la Dalmatie et une zone d'influence en Asie Mineure ; tout cela avant même que les principaux intéressés, les États de la région, aient eu le temps de sortir de la neutralité.

\section{SOUVENIRS DU “FRONT D'ORIENT”}

La politique française dans les Balkans à la fin des années 1930, ses buts, ses méthodes et ses résultats furent le fruit direct du souvenir du "front d'Orient" qui, constitué dans les Balkans dès 1915, contribua largement à donner la victoire à l'Entente en 1918. Pour les diplomates et les militaires français voyant, dès la crise de Munich, se reconstituer un grand ensemble allemand en Europe centrale qu'ils auraient un jour probablement à combattre, il était évident que le conflit n'allait pas se jouer en Occident, où les dispositifs défensifs des lignes Siegfried et Maginot voueraient le front à l'immobilisme, mais bien en Orient d'où dépendait le contact de l'Europe centrale sous contrôle du Reich avec les grandes voies de ravitaillement énergétique. Quelques jours après la conférence de Munich, qui fut moins pour Paris un accord de paix qu'un répit accordé à celle-ci, le chef d'État-major Maurice Gamelin écrivait : " l'Allemagne pourrait reprendre ses visées en Europe orientale. (...) La France doit porter ses efforts en Méditerranée orientale par où s'effectuent les liaisons avec l'Europe centrale "3. Déjà en juillet 1938, l'ambassadeur de France en Grèce, Henri Cosme, avait insisté sur le rôle de Salonique comme base de communication avec la Syrie, l'accès de la mer noire ou des canaux de Suez et de Corinthe et en fin de compte le débouché des pétroles de l'Irak. Il est bien évident que la Méditerranée orientale et particulièrement l'articulation des côtes grecque et turque sont appelées à jouer, à l'occasion d'une guerre européenne, un rôle essentiel pour nous 4

La politique française cherchait donc à nouveau dans la partie méridionale des Balkans, et, à travers elle, sur toute la péninsule, le moyen de prendre

3 Gamelin (Maurice), " Note sur la situation actuelle ", n853, SHAT, dossier 5N579, 12/10/1938.

4 Athènes au Quai d'Orsay 72, MAE, dossier Grèce 203, 11/7/1938. 
l'Allemagne à revers en occupant le débouché naturel de la vallée du Danube vers la Méditerranée et le Levant, de garantir la sécurité des contacts avec l'Union soviétique et le contrôle des voies de ravitaillement énergétique en provenance du Proche-Orient.

Si le côté français était dans des conditions qui rappelaient grandement celles de 1914, il n'en allait pas de même du côté balkanique. En 1914, à la veille de la Grande Guerre, les principaux États balkaniques s'étaient préparés à rentrer de leur propre fait dans le conflit, en fonction des territoires que l'une et l'autre alliance leur offriraient en échange de cette entrée : la Serbie, la Roumanie, puis la Grèce vénizeliste aux côtés de l'Entente, l'Empire ottoman, et la Bulgarie aux côtés des puissances centrales. Rien de tout cela en 1938 : dans toutes les capitales balkaniques, les influences étaient plus que partagées et les gouvernements restaient dans l'indécision en ce qui concernait la position qu'ils prendraient dans le conflit, alors que l'influence économique allemande devenait de plus en plus pesante dans la région 5 . Dans le cas grec, l'exercice de la politique étrangère était partagé entre le roi Georges II, très proche de la Grande Bretagne à qui il devait son retour sur le trône en 1935, d'une part et, d'autre part, le Premier ministre dictateur Ioannis Métaxas, qui n'avait jamais caché son admiration pour une Allemagne autoritaire face aux démocraties occidentales soumises à l'instabilité et l'anarchie ; le pays réussissait à maintenir une fragile ligne médiane entre ces deux tendances a priori contradictoires par une politique fort prudente ${ }^{6}$. Le modèle de cette politique fut incontestablement celui de la Turquie qui, dirigée par Ismet Inönü, continuait à vivre de la vieille pratique ottomane en matière de politique étrangère d'équilibre des puissances que Kemal Ataturk avait repris dans les années 19307. En ce qui concerne la France, Paris pouvait donc s'attendre, à la fin de 1938 , à un total refus de la part des diplomaties balkaniques de s'engager en faveur de l'un ou de l'autre camp.

Les seuls véritables éléments qui pouvaient obliger ces États à quitter la politique des équilibres étaient les ambitions territoriales ouvertes des deux États révisionnistes directement ou indirectement impliqués dans la région : la Bulgarie, qui espérait retrouver son accès thrace à la mer Égée et l'Italie, qui incluait dans ses ambitions expansionnistes, affirmées dès 1935, les vieilles “terres irrédentes" et les zones d'influence en Méditerranée orientale qu'elle avait déjà tenté d'arracher lors du premier conflit mondial. En ce qui concerne la

5 Duroselle (Jean-Baptiste), Politique étrangère de la France. La décadence 1932-1939, Paris : Imprimerie Nationale, 1979 , p. 375.

${ }^{6}$ Kitsikis (Dimitri), " La Grèce entre l'Allemagne et l'Angleterre de 1936 à 1941 ", Revue Historique, 238, été 1967 , pp. 85-116.

7 Oran (Baskin), ed., Türk Dis Politikasi. Cilt I : 1919-1980 [La politique étrangère turque. Tome I : 1919-1980], Istanbul : Ilitesim Yayinlari, 2001, p. 19. 
Bulgarie, les États qui en étaient les voisins menacés espéraient que le Pacte balkanique d'assistance mutuelle signé entre eux en 1934 allait suffire pour écarter la menace sans avoir besoin de demander l'aide de l'extérieur. C'est surtout la politique de Rome qui faisait peser la balance régionale du côté des alliés.

C'est ainsi que se présentait la situation au printemps 1939, lorsque la France mena une politique balkanique visant à la construction d'une alliance militaire qui permettrait la constitution d'un nouveau front d'Orient. L'idée était de faire de cette alliance franco-balkanique le flanc sud d'une grande alliance allant de la mer Baltique à la Méditerranée, incluant la Pologne et l'URSS, et qui permettrait de contrôler les mouvements italiens en Méditerranée, ainsi que de combattre l'Allemagne sur deux grands fronts ouest et est ${ }^{8}$. Le point central du dispositif oriental devait être une importante base à Salonique où, aux dires de Gamelin, le drapeau français devait flotter dès le début des hostilités9.

Pour mener à bien le projet, Paris ne se priva pas d'efforts, en essayant d'abord d'y intéresser la Turquie dont le contrôle sur les détroits était capital pour une alliance incluant l'URSS ; à travers la Turquie on pensait attirer son allié grec $^{10}$ qui offrirait le port de Salonique, puis les deux États de l'Entente balkanique, la Yougoslavie et la Roumanie. La conjoncture apparaissait fort favorable pour le rapprochement de ces États avec la France, étant donné qu'en avril 1939, Mussolini, qui deviendra l'allié officiel du troisième Reich avec la signature du Pacte d'Acier en mai, avait attaqué et annexé l'Albanie. La menace italienne était désormais aux frontières directes, non seulement de la Turquie (Dodécanèse), mais aussi de la Grèce et de la Yougoslavie. Ce changement de décor dans les Balkans n'a pourtant pas suffi à créer en réaction automatique une alliance franco-balkanique. Il a fallu pour Paris se décider à donner des gages et des monnaies d'échange concrètes pour progresser dans cette direction et notamment se résigner à des concessions territoriales. Ankara se vit offrir, en échange de l'abandon de sa politique des équilibres, le Sandjak d'Alexandrette qui appartenait au mandat français de Syrie tout en étant peuplé d'une majorité relative turcophone. Dès le 20 mars 1939, devant la progression spectaculaire de l'influence allemande en Europe centrale, le ministre français des affaires étrangères Georges Bonnet donna à son ambassadeur à

${ }^{8}$ Gamelin (Maurice), " Note sur l'intérêt d'une occupation préventive de Salonique ", SHAT, dossier 2N225, 2/6/1939.

9 " Rapport de la réunion des chefs d'état-major", SHAT, dossier 2N225, 3/6/1939.

${ }^{10}$ Trois traités politiques signés en 1930, 1933 et 1938, ainsi que l'appartenance à l'Entente balkanique unissaient les deux pays. Voir Mourélos (Yannis), Fictions et réalités. La France, la Grèce et la stratégie des opérations périphériques dans le sud-est européen (1939-1940), Salonique : Institut d'Études Balkaniques, 1990, p. 49. 
Ankara René Massigli le feu vert que ce dernier avait en vain demandé depuis plusieurs mois pour engager des négociations, d'abord sur la cession du Sandjak, puis sur la conclusion d'un pacte franco-turc d'assistance mutuelle. La pratique était entièrement nouvelle pour la diplomatie d'une grande puissance vis-à-vis d'un État balkanique : jusque là, et particulièrement pendant la première guerre mondiale, c'était la cession d'un territoire venait éventuellement après l'entrée effective du pays bénéficiaire dans une alliance et non le contraire ${ }^{11}$. Dans les mêmes jours, la France et la Grande Bretagne garantirent unilatéralement les frontières de la Grèce et de la Roumanie, sans pour autant recevoir quelque engagement concret de la part de ces deux pays ${ }^{12}$.

La politique française atteignait néanmoins son but puisque, craignant l'Italie et attirée par l'annexion pacifique d'Alexandrette, la Turquie accepta de recevoir en mai le Général Maxime Weygand pour négocier les bases d'un accord qui autoriserait " les puissances occidentales à débarquer des forces importantes (...) en Thrace, à Dédéagatch ${ }^{13}$ ou Salonique "14. Parallèlement, l'État Major turc s'engagea à intéresser et intégrer dans ces négociations le gouvernement grec, ce qu'il fit en août. Paris n'était cependant pas au bout des exigences formulées par Ankara et dut s'engager sur plusieurs points pour obtenir la signature du traité : la première opération de l'alliance serait, une fois la guerre avec l'Allemagne et Italie enclenchée de mener une offensive contre le Dodécanèse ; l'URSS rentrerait en guerre en même temps que la Turquie, faute de quoi cette dernière pourrait rester neutre ; la France pourvoirait aux besoins financiers et matériels de l'armée turque. Paris dut se soumettre, bon gré mal gré, aux exigences en se retirant d'abord, le 23 juin 1939, du Sandjak d'Alexandrette.

Malgré ces concessions importantes, la France pouvait, lors de la déclaration de guerre le premier septembre, se féliciter d'avoir atteint son but : la constitution d'une alliance franco-turque et, à travers elle, franco-balkanique pour la constitution d'un Front d'Orient. Weygand avait précisé dans le rapport envoyé à Paris sur sa mission à Ankara l'ampleur du succès : “ Dès la signature de cet accord qui équivaudra à une rupture avec l'Allemagne, on est décidé à se

\footnotetext{
${ }^{11}$ On peut citer le cas de la colonie britannique de Chypre que Londres s'engagea à céder à la Grèce en 1915 uniquement si le gouvernement grec abandonnait immédiatement sa neutralité pour se ranger aux côtés de l'Entente. Cet engagement ne fut d'ailleurs jamais tenu, malgré l'entrée effective d'Athènes dans le conflit en 1917. Voir Prévélakis (Constantinos), La Grande Bretagne et l'internationalisation de la question chypriote, Université de Paris IV Sorbonne : mémoire de Maitrise sous la direction du Professeur Georges-Henri Soutou, 1999.
}

${ }^{12}$ Lobit (Général De), " Note succincte sur la Grèce et l'armée hellénique ", 192, SHAT, dossier 7N2876, $15 / 3 / 1940$.

13 Il s'agit du port d'Alexandroupolis en Thrace grecque.

14 " Rapport du Général Weygand sur sa mission à Ankara ", MAE, dossier Massigli 104, 2/5/1939. 
mettre sans désemparer au travail sur la frontière de Thrace qu'il s'agit de rendre inviolable "15. Déjà, Athènes semblait, aux dires de l'attaché militaire français à Athènes, de Lobit, " vouloir quitter sa neutralité et, poussé par les Turcs, se ranger à nos côtés ${ }^{\prime 16}$. Le 8 septembre, le Président Albert Lebrun signa la création du Théâtre d'Opérations en Méditerranée Orientale (T.O.M.O.) basé à Beyrouth et dirigé par le Général Weygand. Le 19 octobre enfin, la Turquie, la France et la Grande Bretagne signèrent un traité d'assistance mutuelle prévoyant les modalités d'entrée de la Turquie dans le conflit aux côtés des alliés et réciproquement ; le traité réaffirmait d'autre part l'obligation pour les Alliés de soutenir l'effort d'armement turc. C'est sans hésitation que les affiches de propagande françaises pouvaient désormais afficher sur un planisphère la Turquie comme un territoire appartenant pleinement à la Grande Alliance, au même titre que les États du Commonwealth britannique.

Une fois la guerre contre le Reich engagée, Paris eut pour but de concrétiser désormais les aspirations au front d'Orient, avant tout par la préparation d'une tête de pont dans les Balkans que Weygand s'empressa de situer en Grèce ; en voyage à Ankara, le chef du TOMO rendit visite à l'ambassadeur grec en Turquie Raphaël Raphaël à qui il rappela que " par nécessité l'issue de la guerre se jouera à nouveau dans les Balkans, d'où l'importance du port de Salonique "17. L'idée du débarquement à Salonique, que l'on espérait cette fois réaliser dans la concertation, fut étudiée en profondeur par Paris et Londres dès les premiers Conseils suprêmes interalliés à l'automne $1939^{18}$, ainsi que dans la correspondance abondante entretenue par Weygand à Beyrouth avec les représentations de la France dans les pays balkaniques et l'État-Major de l'Armée à Vincennes.

\section{LES CONSÉQUENCES DE LA NOUVELLE DONNE}

Les données du conflit avaient pourtant changé. La collusion entre l'Allemagne et l'URSS en Europe orientale d'une part, la neutralité italienne au cours de l'automne 1939 et l'hiver 1940 d'autre part, étaient des nouvelles données totalement inattendues dans le conflit. Malgré la fin du rêve d'un front allant de la Baltique à la Méditerranée, il n'était pas question pour Paris de re-

15 Idem.

${ }^{16}$ Athènes à l'EMA 173, SHAT, dossier 5N579, 18/8/1939.

17 Ankara à YPEX 23200, AYE, dossier 43 (1940), 11/9/1939.

${ }^{18}$ Voir Bédarida (François), La stratégie secrète des Alliés et la drôle de guerre. Le Conseil suprême interallié, septembre 1939-avril 1940, Paris : PFNSP / Éditions du CNRS, 1979. 
mettre en cause le front d'Orient : celui-ci était toujours indispensable non plus pour contenir les ambitions italiennes en Méditerranée mais plutôt pour prendre à revers l'Allemagne et l'URSS en tenaille. En décembre 1939 en effet, alors que la collusion germano-soviétique avait déjà partagé les États baltes et la Pologne en sphères d'influence, le Conseil Suprême Interallié pensa utiliser les Balkans pour y affronter sur un terrain qu'il considérait favorable aux alliés le Reich et l'Union soviétique. Le projet consistait à utiliser d'abord la bienveillance, voire la collaboration active, de la Turquie pour mener des actions contre le Caucase soviétique dont la production pétrolifère allait en partie à l'Allemagne aux termes des accords germano-soviétiques de l'automne 1939 ; Ankara se verrait proposer en échange des bénéfices territoriaux aux dépens de Moscou dans la région. On espérait dans un second temps attirer le conflit vers le sud-est européen où, la base de Salonique aidant, on croyait fermement pouvoir l'emporter ${ }^{19}$.

L'Italie, quant à elle, paraissait, de même qu'au début de la Grande Guerre, dans une neutralité qui cachait son indécision quant à sa position dans la guerre. Paris et Londres allaient donc désormais tout faire pour ménager Rome dans l'espoir de la garder neutre, voire de l'intégrer dans leur alliance. Le caractère anti-italien du front d'Orient, base pourtant du traité d'alliance avec la Turquie, disparut donc complètement des discussions au sein des états-majors. Un nouveau pas fut franchi en décembre 1939, lorsque la Grande Bretagne ouvrit à Tirana un consulat accrédité auprès des autorités italiennes, reconnaissant ainsi ouvertement l'annexion de l'Albanie par Mussolini. Les messages hésitants arrivant de Rome paraissant encourageants, on projeta à la fin de 1939 de faire de l'Italie le partenaire essentiel du front d'Orient dont la nouvelle forme fut, en tenant compte des nouvelles données du conflit, présentée dans une note du Cabinet du Ministre de la Défense et du président du Conseil Edouard Daladier le 30 décembre 1939 : " Il paraît possible de faire naître un front commun italo-balkanique pour lutter contre une invasion germanorusse. La part des franco-britanniques se réduirait à soutenir les Grecs à partir de la Thrace ou de Salonique "20. La politique française chercha au début de l'année 1940 les moyens par lesquels elle pourrait atteindre son but d'inclure l'Italie dans son front d'Orient. L'ambassade de France à Rome préconisa que dans la presse la plus officieuse, les chefs de gouvernement eux-mêmes abordent, un jour, la question du front et saisissent la première occasion de rappeler (...) qu'ils demeurent disposés à tout échange de vues qui aurait pour but

19 Pour les projets caucasiens de l'état-major français, voir Richardson (Charles O.), "French plans for allied attacks on the Caucasus oil fields, January-April 1940 ", French Historical Studies, 8 (1), printemps 1975 , ainsi que Chassin (Général L.M.), "Un plan grandiose : l'attaque des pétroles du Caucase en 1940 ", Forces aériennes françaises, décembre 1961.

20 " Note sur la conduite stratégique de la guerre ", SHAT, dossier 5N580, 30/12/1939. 
d'établir un statut méditerranéen dont l'Italie put s'estimer satisfaite (...). L'on doit se rappeler aussi que l'on a jamais rien pour rien et que (...) quand nous avons, nous-mêmes, voulu nous attacher les Turcs, nous leur avons cédé le Sandjak d'Alexandrette ${ }^{21}$.

Tout au long de ces bouleversements de tactique, la politique française ne sembla pas comprendre que ce changement d'attitude était de nature à casser le fragile lien de confiance laborieusement établi en 1939 entre la France, la Grèce et surtout la Turquie. La nouvelle politique vis-à-vis de l'Union soviétique, que jamais Athènes et surtout Ankara n'avaient imaginé devoir combattre, et de l'Italie, qui devait être la cible et non le partenaire de l'alliance, fit craindre aux milieux diplomatiques et militaires balkaniques que le scénario de la Première Guerre mondiale allait se répéter et que, pris dans des engagements contradictoires, leurs pays allaient faire les frais de l'antagonisme entre les grandes puissances.

En janvier 1940, l'Ambassadeur grec en France Nikolas Politis tirait, dans son mémoire de fin de mission (et de fin de carrière) au Premier Ministre Métaxas, la sonnette d'alarme :

Malgré le profond respect et l'amour sincère que je nourris pour la France et la Grande Bretagne, ma confiance en leur jugement politique est plus que réduite quand il s'agit du sort de notre patrie (...). Dans la presse britannique et française on parle souvent des intérêts vitaux de l'Italie dans les Balkans. Le seul contrepoids sûr et durable est (...) de résoudre les différends inter-balkaniques pour éviter autant que possible l'intervention des grandes puissances.

À défaut de cette résolution, qui, dans l'esprit de Politis, passait par la satisfaction de certaines revendications territoriales de la Bulgarie en Thrace et en Dobroudja afin de pouvoir élargir l'Entente balkanique à l'ensemble de la péninsule, l'Ambassadeur recommandait comme solution de rechange pour son pays vis-à-vis de la France " d'obtenir le titre et la place de l'Allié afin de se protéger de l'éventuelle manifestation à ses dépens de l'égoïsme naturel des Grandes Puissances lors de la mise en oeuvre de la nouvelle carte de l'Europe "22. Venant de Politis, le discours est surprenant. Cette personnalité fort influente dans la réflexion diplomatique en Grèce dès les années 1910 avait lié son nom au parti "francophile" grec : étudiant en France, reçu premier à l'Agrégation de Droit, Professeur de Droit international aux Universités de Poitiers puis de Paris, Politis fut en 1917 le ministre des Affaires Étrangères du gouvernement Vénizelos qui, porté à Athènes par une intervention militaire française contre le roi Constantin $\mathrm{I}^{\mathrm{er}}$, engagea sans réserve la Grèce dans la guerre aux côtés de l'Entente. Politis avait alors suivi la politique aventurière

\footnotetext{
${ }^{21}$ Rome au Quai d'Orsay 1852, MAE, dossier Massigli 22, 25/3/1940.

${ }^{22}$ Paris à YPEX 2661, AYE, dossier 7.1, 16/1/1940.
} 
de Vénizelos qui consistait à s'engager ouvertement aux côtés de la France dans l'espoir d'obtenir des compensations territoriales, aux dépens principalement de l'Empire ottoman et de la Bulgarie. Vingt ans plus tard, la même personnalité terminait sa carrière à l'Ambassade de la rue Auguste Vacquerie en proposant des concessions territoriales à la Bulgarie pour éviter une alliance avec la France !

Le résultat de plus en plus marqué, dans les premiers mois de 1940, de cette vision de la situation fut l'adoption de la part de la Grèce et de la Turquie d'une politique attentiste dont le but inavoué était de se désengager autant que possible d'une alliance dont on pensait que, loin d'être un rempart contre la menace italienne que Paris et Londres semblaient désormais ménager, elle ne pourrait que faciliter l'agression allemande ou soviétique. En avril l'issue des opérations des Alliés en Norvège qui s'est soldée par une invasion-éclair allemande ne fit qu'aggraver cette crise de confiance. Le 5 mai 1940, un état de l'opinion publique turque rédigé par le consulat français de Zongouldak affirmait que "les esprits indigènes commencent en somme à se demander si leurs gouvernements ont fait le meilleur choix et, surtout, s'il était vraiment opportun de prendre parti "23. Le Général Weygand s'était lui-même, le 19 avril, plaint dans un message envoyé depuis Beyrouth à René Massigli : “Les Turcs ont été jusqu'ici bien peu opérants. Ils s'étaient chargés de parler aux Grecs et n'en ont rien fait. Quant aux Balkaniques, les ont-ils seulement tâtés ? (...) Ces amis veulent que nous intervenions instantanément et massivement (...) mais sans avoir fait eux-mêmes un pas, risqué un accord secret qui faciliterait cette intervention "24. Le 9 mai, le Général trouva que les Turcs " sont restés de certains côtés terriblement orientaux. Le temps ne compte pas "25. Cette appréciation, à la limite du cliché, est surprenante de la part de Weygand, quand on sait que, dans son précédent message à Massigli, il avait lui-même localisé précisément le fond du problème franco-balkanique : "Le problème est insoluble. Nous tournons en rond. Disons-le court, il faut que les Balkaniques prennent parti et pour y aboutir, il faut que nous leur donnions confiance, ce qu'ils n'ont pas " 26 . Rétablir la confiance ? Trop tard sans doute à la fin avril, alors que le dix mai les troupes allemandes envahissaient la France, et que, contre toute attente, le front d'Occident théoriquement voué à l'immobilisme succombait à la Blitzkrieg en six semaines.

\footnotetext{
${ }^{23}$ Zongouldak à Ankara 58-59, MAE, dossier Massigli 23, 5/5/1940.

24 Weygand à Massigli, MAE, dossier Massigli 104, 24/4/1940.

25 Ibid., 9/5/1940.

${ }^{26}$ Ibid., 24/4/1940.
} 
Qu'en conclure ? D'abord, que les Balkans furent de par leur situation incontournables dans l'élaboration d'une stratégie à l'échelle européenne et le Quai d'Orsay eut la présence d'esprit de s'en rendre compte dès 1938. Ensuite, que l'intégration des États balkaniques dans un système européen d'alliances dominé par la France fut bien plus qu'une affaire de concessions territoriales et de garanties. Il dépendait d'abord des buts et de la structure de l'alliance qui, incontestablement, façonnaient le paysage géopolitique balkanique. Paris a mis du temps à comprendre qu'utiliser une alliance conçue pour collaborer avec Moscou contre Rome afin de prendre à revers l'URSS avec l'aide de l'Italie était inconcevable pour Ankara, Athènes et Belgrade.

Surtout, et ceci découle aussi du changement de tactique opéré par la France à l'automne 1939, l'affaire balkanique était une affaire de confiance ; et la confiance pourrait ici être entendue comme une des "forces profondes" des relations internationales pour reprendre le terme de Pierre Renouvin ${ }^{27}$. C'est cette confiance que la diplomatie et l'état-major français pensaient avoir acquise par des méthodes du début du XX ${ }^{\text {ème }}$ siècle. Voulant retenir les leçons de la Grande Guerre, Paris a quelque peu révisé ces méthodes en faisant des concessions territoriales et des engagements solennels des préalables et non plus des résultats de l'alliance franco-balkanique. Or en 1940, les capitales balkaniques avaient de même retenu ces leçons, mais à une échelle tout autre que les grandes puissances. Cette différence d'échelle dans l'estimation de la solidité et la fiabilité des engagements réciproquement donnés fut la base de la crise de confiance qui mena la politique française à l'échec.

L'Europe vit actuellement en paix et les rapports de force inter-européens ne se jouent certes plus comme en 1940. Cependant, au moment où l'on parle d'européaniser les Balkans, c'est-à-dire de les intégrer dans un grand ordre européen qui leur garantirait la prospérité et surtout la sécurité, au moment où l'on pense que des élites balkaniques "occidentalisées" peuvent facilement rentrer et demeurer dans un rapport de confiance avec les grandes puissances, la leçon balkanique de 1938-1940 constitue peut-être un des précieux services que l'Histoire puisse rendre à la science politique.

${ }^{27}$ Renouvin (Pierre), Duroselle (Jean-Baptiste), Introduction à l'histoire des relations internationales, Paris : Armand Colin, 1991 [4e édition]. 